\title{
Erratum: Phonons, magnons, and lattice thermal transport in antiferromagnetic semiconductor MnTe [Phys. Rev. Materials 3, 025403 (2019)]
}

\author{
Sai Mu, Raphaël P. Hermann, Stéphane Gorsse, Huaizhou Zhao, Michael E. Manley, Randy S. Fishman, and L. Lindsay
}

(Received 28 February 2019; published 22 March 2019)

DOI: 10.1103/PhysRevMaterials.3.039901

One sentence in the caption of Table I is rephrased, and several references are modified. These modifcations do not affect the results, discussions, or the conclusions of the paper.

(1) To avoid confusion, we rephrase "As defined, negative $J_{n}$ indicates the antiferromagnetic coupling" in the caption of Table I to "As defined in Eq. (3), the presented $J_{n}$ is the exchange interaction between normalized spins, and negative $J_{n}$ indicates the antiferromagnetic coupling."

(2) The description of local moments of previous work (on p. 4) should read: "Employing $U_{\text {eff }}=4.8 \mathrm{eV}$, the calculated local moment on $\mathrm{Mn}$ sites in the ground state is about $4.55 \mu_{\mathrm{B}}$, consistent with previous work: $4.76 \mu_{B}$ [9], 4.66 $\mu_{B}$ [38], 4.27 $\mu_{B}$ [14], $4.52 \mu_{B}[13] . "$

(3) The reference regarding magnetic susceptibility measurements (on p. 5) should read: "Magnetic susceptibility measurements [48] of $\alpha$-MnTe give a Curie-Weiss temperature ...."

(4) The caption of Fig. 3(a) (on p. 5) should read: "Measured data are also shown for magnons at $T=11 \mathrm{~K}$ (blue circles) [9], an $M$-point magnon at $T=300 \mathrm{~K}$ (blue triangle), and phonons at $T=300 \mathrm{~K}$ (black circles) [49]."

The corresponding discussion on the same page should read: "Figure 3(a) gives the calculated spin-wave (magnon) dispersion for the antiferromagnetic state of $\alpha$-MnTe compared with measured dispersion data at $T=11 \mathrm{~K}$ [9] and a single data point at $T=300 \mathrm{~K}$ [49]. Also given are the calculated and measured [49] phonon dispersions."

[9] W. Szuszkiewicz, E. Dynowska, B. Witkowska, and B. Hennion, Phys. Rev. B 73, 104403 (2006).

[13] M. Krause and F. Bechstedt, J. Supercond. Nov. Magn. 26, 1963 (2013).

[14] D. Kriegner, H. Reichlova, J. Grenzer, W. Schmidt, E. Ressouche, J. Godinho, T. Wagner, S. Y. Martin, A. B. Shick, V. V. Volobuev, G. Springholz, V. Holý, J. Wunderlich, T. Jungwirth, and K. Výborný, Phys. Rev. B 96, 214418 (2017).

[38] S. J. Youn, B. I. Min, and A. J. Freeman, Phys. Status Solidi B 241, 1411 (2004).

[48] T. Komatsubara, M. Murakami, and E. Hirahara, J. Phys. Soc. Japan 18, 356 (1963).

[49] W. Szuszkiewicz, B. Hennion, B. Witkowska, E. Łusakowska, and A. Mycielski, Phys. Status Solidi C 2, 1141 (2005). 